\title{
On Moments of Gamma-Exponentiated Functional Distribution
}

\author{
Katarzyna Górska ${ }^{1}$, Andrzej Horzela ${ }^{2}$ and Tibor K. Pogány ${ }^{3, *}$ \\ 1 H. Niewodniczański Institute of Nuclear Physics, Polish Academy of Sciences, Division of Theoretical Physics, \\ ul. Eliasza-Radzikowskiego 152, PL 31-342 Kraków, Poland; katarzyna.gorska@ifj.edu.pl \\ 2 Faculty of Maritime Studies, University of Rijeka, Studentska 2, HR-51000 Rijeka, Croatia; andrzej.horzela@ifj.edu.pl \\ 3 Institute of Applied Mathematics, Óbuda University, Bécsi út 96/b, H-1034 Budapest, Hungary \\ * Correspondence: poganj@pfri.hr
}

Received: 24 February 2018; Accepted: 27 March 2018; Published: 30 March 2018

check for updates

\begin{abstract}
In this note we discuss the development of a new Gamma exponentiated functional GE $(\alpha, h)$ distribution, using the Gamma baseline distribution generating method by Zografos and Balakrishnan. The raw moments of the Gamma exponentiated functional $\mathrm{GE}(\alpha, h)$ distribution are derived. The related probability distribution class is characterized in terms of Lambert $W$-function.
\end{abstract}

Keywords: Gamma-exponentiated functional distribution; moments; Lagrange-Bürmann inversion theorem; Lambert $W$-function; quantile function

MSC: 60E05, 62E15, 33C20

\section{Introduction}

The idea of adding a positive parameter in the exponent of the cumulative distribution function (cdf) for a continuous distribution was introduced by Lehmann [1], which results in a class of so-called exponentiated distribution, see also ([2], Chapter 2). Using exponentiated Gamma-type random variables (rv) (or Stacy's generalized Gamma distribution [3]) for generating further distribution classes were used by Zografos and Balakrishnan ([4], p. 350 et seq.). Subsequently, considering a similar method Ristić and Balakrishnan ([5], p. 1192, Equation (2)) introduce a new family od distributions via the survival function of the general continuous baseline (or parent) distribution which turns out to be a "dual family of the Zografos-Balakrishnan family of distributions" ([5], p. 1192) with a set of three main motivations for their new distribution class [5], ibid. We point out that Ristic and Balakrishnan linked their study to the Gamma-exponentiated exponential distribution, which certain properties are discussed by Ristić and Nadarajah [6]; it is worth mentioning the companion note by Pogány [7] where their findings concerning moments are precised in terms of higher transcendental functions including confluent Fox-Wright generalized hypergeometric and generalized Hurwitz-Lerch zeta function.

Introducing two extra parameters Cordeiro et al. ([8], pp. 1-2) have covered both Lehmann I and Lehmann II type exponentiated distribution classes, calling these exponentiated generalized families, giving full consideration to certain special cases like exponentiated generalized Frèchet, Normal, Gamma and Gumbel distributions ([8], 2. Special Models). Numerous authors have linked to listed models considering special cases of Gamma generalized, exponentiated distribution classes, among others we refer to Gamma-exponentiated Weibull [9,10], exponentiated Weibull, exponentiated Pareto, exponentiated Gamma [11], Kumaraswamy generalized Gamma and Gumbel [12,13] distributions with exhaustive references lists and links to further sub-models and special cases, consult e.g., ([13], pp. 415-416); also see the recent article [14] where an extension is obtained for the generalized integro-exponential function 
by which the moment expression of the above listed distribution classes can be expressed in a closed or more compact form. Finally, we mention the related recent article [15] as well.

The main purpose of adding parameters to an existing distribution is to obtain classes of more flexible distributions which provide more adaptability in modeling various types of data. According to Zografos and Balakrishnan [4] the Gamma-exponentiated extended distribution possesses cdf $F(x)$ given as

$$
F(x)=\frac{1}{\Gamma(\alpha)} \int_{0}^{-\log \bar{G}(x)} t^{\alpha-1} \mathrm{e}^{-t} \mathrm{~d} t, \quad \alpha>0, x \in \mathbb{R},
$$

where the baseline distribution $G$ has the survival function $\bar{G}(x)=1-G(x)$. The Gamma-exponentiated extended probability density function (pdf) related to Equation (1) can be expressed in the following form:

$$
f(x)=\frac{1}{\Gamma(\alpha)}[-\log \bar{G}(x)]^{\alpha-1} G^{\prime}(x), \quad \alpha>0, x \in \mathbb{R} .
$$

The regularized Gamma function reads

$$
Q(a, z)=\frac{\Gamma(a, z)}{\Gamma(a)}=\frac{1}{\Gamma(a)} \int_{z}^{\infty} t^{a-1} \mathrm{e}^{-t} \mathrm{~d} t, \quad \Re(a)>0,
$$

where $\Gamma(a, x)$ denotes the upper incomplete Gamma function. Both, regularized Gamma and incomplete Gamma, are in-built in Mathematica under GammaRegularized [a, z] and Gamma [a, z], respectively.

We specify the approach presented in [4] by choosing the baseline distribution's survival function to be $\bar{G}(x)=1-G(x)=\exp (-h(x))$, where $h: \mathbb{R}_{+} \mapsto \mathbb{R}_{+}$denotes a nonnegative Borel function.

The rv $X$ defined on a standard probability space $(\Omega, \mathfrak{F}, \mathrm{P})$, having $\mathrm{cdf}$ and pdf

$$
\begin{aligned}
& F(x)=[1-Q(\alpha, h(x))] 1_{\mathbb{R}_{+}}(x) \\
& f(x)=\frac{h^{\prime}(x)}{\Gamma(\alpha)} h^{\alpha-1}(x) \mathrm{e}^{-h(x)} 1_{\mathbb{R}_{+}}(x),
\end{aligned}
$$

respectively, is called Gamma-exponentiated functional $h$ distributed, signifying this $X \sim \operatorname{GE}(\alpha, h)$. Here and in what follows, $1_{A}(x)$ denotes the indicator function of the set $A$, i.e., $1_{A}(x)=1$ when $x \in A$ and equals 0 elsewhere.

As an illustrative example of this approach can be the case considered by Pogány and Saboor [16] choosing $h(x)=\lambda x+\beta x^{k}$ introduced the Gamma-exponentiated exponential Weibull distribution $\operatorname{GEEW}(\theta), \theta=(\lambda, \beta, k, \alpha)>0$, which cdf and pdf are

$$
\begin{aligned}
& F(x)=\left[1-Q\left(\alpha, \lambda x+\beta x^{k}\right)\right] 1_{\mathbb{R}_{+}}(x) \\
& f(x)=\frac{1}{\Gamma(\alpha)}\left(\lambda+\beta k x^{k-1}\right) \mathrm{e}^{-\lambda x-\beta x^{k}}\left(\lambda x+\beta x^{k}\right)^{\alpha-1} 1_{\mathbb{R}_{+}}(x) .
\end{aligned}
$$

Finally, the incomplete Gamma function possesses a representation in terms of the Kummer's confluent hypergeometric function ([17], Chapter 13)

$$
{ }_{1} F_{1}(a ; b ; z)=\sum_{n \geq 0} \frac{(a)_{n}}{(b)_{n}} \frac{z^{n}}{n !},
$$

we have the equivalent form of the cdf

$$
F(x)=\frac{h^{\alpha}(x)}{\Gamma(\alpha+1)}{ }_{1} F_{1}(\alpha ; \alpha+1 ;-h(x)) ;
$$


the particular cases of ${ }_{1} F_{1}$ to other functions as elementary, incomplete Gamma, orthogonal polynomials, generalized hypergeometric, Bessel, Coulomb functions are documented e.g., in [17], §13.6. In this note, bearing in mind this relationship and motivated with the general model of distribution families by Zografos and Balakrishan, and also the model by Ristić and Balakrishnan which can be described by the Gamma exponentiated functional model GE $(\alpha, h)$ we derive two formulae for the raw moments of $\mathrm{rv}$ having $\operatorname{GE}(\alpha, h)$ distribution in terms of a hypergeometric function the integrand. Moreover, the random variable $X \sim \operatorname{GE}(\alpha, h)$ has been characterized and described in terms of the Lambert's $W$ function.

\section{Moments and Quantile Function}

Firstly, we note that further on ${ }_{p} F_{q}$ denotes the generalized hypergeometric function of $p$ numerator and $q$ denominator parameters, see e.g., [18]; for ${ }_{2} F_{0}$ we refer to [19].

Theorem 1. Let $h: \mathbb{R}_{+} \mapsto \mathbb{R}_{+}$be analytic monotone increasing function with $h^{\prime}(0) \neq 0$ and let $r v X \sim \operatorname{GE}(\alpha, h)$. Then for all $\alpha>0, r \geq 0$ we have

$$
\mathrm{E} X^{r}=\frac{r}{4 \pi^{2}} \oint_{\gamma_{z}} \oint_{\gamma_{w}} \frac{z^{r-1}\left[(1-w)^{\alpha}-1\right]}{h(z) w^{2}(1-w)^{\alpha}}{ }_{2} F_{0}\left(1,1 ;-; \frac{1}{h(z) w}\right) \mathrm{d} z \mathrm{~d} w,
$$

where the positively oriented closed integration paths $\gamma_{z}, \gamma_{w}$ are taken in a way that enclose the origins in the complex $z-$, and $w$-planes, respectively.

Moreover, under the same assumptions, we have

$$
\mathrm{E} X^{r}=\frac{r}{2 \pi \mathrm{i}} \oint_{\gamma_{z}} \int_{0}^{\infty} \frac{z^{r-1}\left[(1-t)^{-\alpha}-1\right]}{t} \mathrm{e}^{-h(z) t} \mathrm{~d} z \mathrm{~d} t
$$

Proof. Assume $r>0$ and denoting $h^{-1}$ the inverse of $h$, we have

$$
\mathrm{E} X^{r}=\int_{0}^{\infty} \frac{x^{r}}{\Gamma(\alpha)} h^{\prime}(x) h^{\alpha-1}(x) \mathrm{e}^{-h(x)} \mathrm{d} x=\frac{1}{\Gamma(\alpha)} \int_{0}^{\infty}\left[h^{-1}(t)\right]^{r} t^{\alpha-1} \mathrm{e}^{-t} \mathrm{~d} t
$$

The Lagrange-Bürmann inversion theorem ([20], Equation (1.1) et seq.) reads:

Let $a(z)=\sum_{n \geq 0} a_{n} z^{n}$, with $a_{1} \neq 0$ (interpreted either as analytic function or a formal power series), and $A(z)=\sum_{n \geq 0} A_{n} z^{n}$. Then

$$
A\left(a^{-1}(z)\right)=A_{0}+\sum_{n \geq 1} \frac{z^{n}}{n}\left[\zeta^{n-1}\right] A^{\prime}(\zeta)\left(\frac{\zeta}{a(\zeta)}\right)^{n}
$$

where $\left[\zeta^{m}\right]$ extracts the coefficient of $\zeta^{m}$ in a series: $\left[\zeta^{m}\right]\left(\sum_{k} c_{k} x^{k}\right)=c_{m}$.

Applying Equation (5) to the integrand of the moment $\mathrm{E} X^{r}$ above, being $a \equiv h$ and $A(\zeta)=\zeta^{r}, r \geq 0$, we conclude

$$
\begin{aligned}
\mathrm{E} X^{r} & =\frac{r}{\Gamma(\alpha)} \sum_{n \geq 1} \frac{1}{n}\left(\int_{0}^{\infty} t^{\alpha+n-1} e^{-t} \mathrm{~d} t\right)\left[\zeta^{n-1}\right] \frac{\zeta^{r+n-1}}{h^{n}(\zeta)} \\
& =r \sum_{n \geq 1} \frac{(\alpha)_{n}}{n}\left[\zeta^{n-1}\right] \frac{\zeta^{r+n-1}}{h^{n}(\zeta)} \\
& =r \alpha \sum_{n \geq 0} \frac{(\alpha+1)_{n}(1)_{n}}{(2)_{n}}\left[\zeta^{n}\right] \frac{\zeta^{r+n}}{h^{n+1}(\zeta)}
\end{aligned}
$$

By the Cauchy differentiation formula we have 


$$
\left[\zeta^{n}\right] \frac{\zeta^{r+n}}{h^{n+1}(\zeta)}=\left.\frac{1}{n !} \frac{\mathrm{d}^{n}}{\mathrm{~d} \zeta^{n}}\left(\frac{\zeta^{r+n}}{h^{n+1}(\zeta)}\right)\right|_{\zeta=0}=\frac{1}{2 \pi \mathrm{i}} \oint_{\gamma_{z}} \frac{z^{r-1}}{h^{n+1}(z)} \mathrm{d} z
$$

that is

$$
\mathrm{E} X^{r}=\frac{r \alpha}{2 \pi \mathrm{i}} \oint_{\gamma_{z}} \frac{z^{r-1}}{h(z)} \sum_{n \geq 0} \frac{(\alpha+1)_{n}(1)_{n}}{(2)_{n}} \frac{1}{h^{n}(z)} \mathrm{d} z,
$$

where $\gamma_{z}$ is a positively oriented simple integration path enclosing the origin. Having in mind the differentiation property of the Gauss' hypergeometric function ([18], p. 28, (1.6.11))

$$
\left.\frac{\partial^{n}}{\partial w^{n}}{ }_{2} F_{1}(a, b ; c ; w)\right|_{w=0}=\frac{(a)_{n}(b)_{n}}{(c)_{n}}{ }_{2} F_{1}(a+n, b+n ; c+n ; 0)=\frac{(a)_{n}(b)_{n}}{(c)_{n}},
$$

it follows also by the Cauchy's differentiation formula:

$$
\begin{aligned}
\frac{(\alpha+1)_{n}(1)_{n}}{(2)_{n}} & =\left.\frac{\partial^{n}}{\partial w^{n}}{ }_{2} F_{1}(\alpha+1,1 ; 2 ; w)\right|_{w=0} \\
& =\frac{n !}{2 \pi \mathrm{i}} \oint_{\gamma_{w}} \frac{{ }_{2} F_{1}(\alpha+1,1 ; 2 ; w)}{w^{n+1}} \mathrm{~d} w .
\end{aligned}
$$

Choosing the integration paths $\gamma_{z}, \gamma_{w}$ according to the assumptions we get

$$
\mathrm{E} X^{r}=-\frac{r \alpha}{4 \pi^{2}} \oint_{\gamma_{z}} \oint_{\gamma_{w}} \frac{z^{r-1}{ }_{2} F_{1}(\alpha+1,1 ; 2 ; w)}{h(z) w} \sum_{n \geq 0} \frac{(1)_{n}}{(h(z) w)^{n}} \mathrm{~d} z \mathrm{~d} w
$$

which is in fact Equation (3) since

$$
{ }_{2} F_{1}(\alpha+1,1 ; 2 ; w)=-\frac{(1-w)^{\alpha}-1}{\alpha w(1-w)^{\alpha}} .
$$

The rest is obvious.

As to Equation (4), we take the Laplace-integral formula ([18], p. 31, Equation (1.6.33)):

$$
{ }_{3} F_{1}\left(\lambda, a, b ; c ; s^{-1}\right)=\frac{s^{\lambda}}{\Gamma(\lambda)} \int_{0}^{\infty} \mathrm{e}^{-s t} t^{\lambda-1}{ }_{2} F_{1}(a, b ; c ; t) \mathrm{d} t,
$$

which holds true for all $a, b \in \mathbb{C} ; c \in \mathbb{C} \backslash \mathbb{Z}_{-}^{0}$ provided that $\min \{\Re(\lambda), \Re(s)\}>0$. Thus, starting from Equation (6), we transform the inner sum into a ${ }_{3} F_{1}$ expression by Equation (8) and conclude

$$
\begin{aligned}
\mathrm{EX}^{r} & =\frac{r \alpha}{2 \pi \mathrm{i}} \oint_{\gamma_{z}} \frac{z^{r-1}}{h(z)} \sum_{n \geq 0} \frac{(1)_{n}(\alpha+1)_{n}(1)_{n}}{(2)_{n} n !}\left(\frac{1}{h(z)}\right)^{n} \mathrm{~d} z \\
& =\frac{r \alpha}{2 \pi \mathrm{i}} \oint_{\gamma_{z}} \frac{z^{r-1}}{h(z)}{ }_{3} F_{1}\left(1, \alpha+1,1 ; 2 ; \frac{1}{h(z)}\right) \mathrm{d} z \\
& =\frac{r \alpha}{2 \pi \mathrm{i}} \oint_{\gamma_{z}} z^{r-1} \int_{0}^{\infty} \mathrm{e}^{-h(z) t}{ }_{2} F_{1}(\alpha+1,1 ; 2 ; t) \mathrm{d} z \mathrm{~d} t .
\end{aligned}
$$

By Equation (7), the expression Equation (9) becomes

$$
\mathrm{E} X^{r}=\frac{r}{2 \pi \mathrm{i}} \oint_{\gamma_{z}} \int_{0}^{\infty} \frac{z^{r-1}\left[(1-t)^{-\alpha}-1\right]}{t} \mathrm{e}^{-h(z) t} \mathrm{~d} z \mathrm{~d} t
$$

which completes the proof.

The consequence of Theorem 1 when $r=1$, recalling that $\mathrm{E} X=\alpha$, is 
Corollary 1. For all $\alpha>0$ we have

$$
\begin{array}{r}
\oint_{\gamma_{z}} \oint_{\gamma_{w}} \frac{(1-w)^{\alpha}-1}{z w^{2}(1-w)^{\alpha}}{ }_{2} F_{0}\left(1,1 ;-; \frac{1}{z w}\right) \mathrm{d} z \mathrm{~d} w=4 \pi^{2} \alpha \\
\oint_{\gamma_{z}} \int_{0}^{\infty} \frac{\left[(1-t)^{-\alpha}-1\right]}{t} \mathrm{e}^{-z t} \mathrm{~d} z \mathrm{~d} t=2 \pi \mathrm{i} \alpha .
\end{array}
$$

Here the integration contours $\gamma_{z}, \gamma_{w}$ remain the same as in Theorem 1.

The Lambert $W$-function is the inverse function of $W \mapsto W \mathrm{e}^{W}$. Its principal branch $W_{\mathrm{P}}$ is the solution of $W \mathrm{e}^{W}=x$, for which $W_{\mathrm{P}}(x) \geq W_{\mathrm{P}}\left(-\mathrm{e}^{-1}\right)$. This function is in-built in Mathematica as ProductLog $[\mathrm{z}]$. We are interested in $W_{\mathrm{P}}$ exclusively for $x \geq 0$, where it is single-valued and monotone increasing, see [17], Section 4.13 .

Any nondecreasing function $h$ possesses an generalized inverse

$$
\mathfrak{h}^{-}(x):=\inf \left\{t \in \mathbb{R}_{+}: h(t) \geq x\right\}, \quad t \in \mathbb{R}_{+},
$$

with the convention that inf $\varnothing=\infty$. Moreover, if $h$ is strong monotone increasing then $\mathfrak{h}^{-}$coincides with the 'ordinary' inverse $h^{-1}$.

Theorem 2. Consider rv $\mathrm{Y}=h^{p}(X) \exp (\sigma h(X)) ; \sigma, p \geq 0$, where $\mathrm{X} \sim \mathrm{GE}(\alpha, h)$. Then

$$
\mathrm{Y} \sim \mathrm{GE}\left(\alpha, \mathfrak{h}^{-}\right), \quad \mathfrak{h}^{-}:=\mathfrak{h}^{-}\left[\frac{p}{\sigma} W_{\mathrm{P}}\left(\frac{\sigma}{p} x^{\frac{1}{p}}\right)\right] .
$$

Moreover, for all $s \in\left(-\alpha p^{-1}, \sigma^{-1}\right)$ we have

$$
\mathrm{EY}^{s}=\frac{(\alpha)_{p s}}{(1-\sigma s)^{\alpha+s p}}
$$

whenever $h: \mathbb{R}_{+} \mapsto \mathbb{R}_{+}$is a nondecreasing Borel function.

Proof. The rv $X \sim \operatorname{GE}(\alpha, h)$ possesses cdf $F_{X}$ in the form Equation (2). When $\sigma=0$, then $Y \equiv h^{p}(X)$. Letting $\sigma>0$, the pdf $F_{Y}$ of the rv Y becomes

$$
\begin{aligned}
F_{Y}(x) & =\mathrm{P}\left[h(X) \exp \left(\frac{\sigma}{p} h(X)\right)<x^{\frac{1}{p}}\right]=\mathrm{P}\left[h(X)<\frac{p}{\sigma} W_{\mathrm{P}}\left(\frac{\sigma}{p} x^{\frac{1}{p}}\right)\right] \\
& =\mathrm{P}\left\{X<\mathfrak{h}^{-}\left[\frac{p}{\sigma} W_{\mathrm{P}}\left(\frac{\sigma}{p} x^{\frac{1}{p}}\right)\right]\right\}=F_{X}\left\{\mathfrak{h}^{-}\left[\frac{p}{\sigma} W_{\mathrm{P}}\left(\frac{\sigma}{p} x^{\frac{1}{p}}\right)\right]\right\} \cdot 1_{\mathbb{R}_{+}}(x),
\end{aligned}
$$

which is equivalent to the first assertion Equation (10). In turn

$$
\mathrm{EY}^{s}=\mathrm{E} h^{p s}(X) \exp (\sigma s h(X))=\frac{1}{\Gamma(\alpha)} \int_{0}^{\infty} h^{\alpha+p s-1}(x) \mathrm{e}^{-(1-\sigma s) h(x)} \mathrm{d} h(x)
$$

where the convergence of the integral is controlled by the condition $\sigma s<1$ because $h$ is non-decreasing and positive at the infinity. Now, routine steps lead to the assertion.

The quantile function $\mathscr{Q}_{X}$ of the $\operatorname{rv} X \sim F(x)$ is defined as

$$
\mathscr{Q}_{X}(p)=\inf \{x \in \mathbb{R}: p \leq F(x)\}, \quad p \in(0,1) .
$$

It is the generalized inverse of the cdf for a fixed probability $p$. The related result is the following 
Theorem 3. Let $X \sim \mathrm{GE}(h)$, where $h: \mathbb{R}_{+} \mapsto \mathbb{R}_{+}$is a nonnegative monotone Borel function. Then the quantile function $\mathscr{Q}_{X}(p)$ reads

$$
\mathscr{Q}_{X}(p)=h^{-1} \circ Q^{-1}(\alpha, 1-p), \quad p \in(0,1),
$$

where $\circ$ denotes the composition of functions.

Proof. The quantile function is derived by inverting Equation (2). Therefore, for $p \in(0,1)$ fixed, solving the equation $1-Q(\alpha, h(x))=F(x)=p$ with respect to the regularized upper incomplete Gamma-function $Q$, we get $Q(\alpha, h(x))=1-p$. Because $\Gamma^{\prime}(a, z)=-z^{a-1} \mathrm{e}^{-z}<0$, the function $\Gamma(a, z)=\Gamma(\alpha) Q(a, z)$ is monotone in $z$, therefore $Q$ has an unique inverse $Q^{-1}$ :

$$
h(x)=Q^{-1}(\alpha, 1-p)
$$

Remarking that $h$ is monotone too, the proof is finished.

\section{Concluding Remarks}

In this manuscript, the authors discuss the development of a new distribution, Gamma exponentiated functional GE $(\alpha, h)$ distribution, using the Gamma baseline distribution generating method by Zografos and Balakrishnan [4] and also related to the so called "dual family of the Zografos-Balakrishnan family of distributions" [5]. The main findings of the article are two equaivalent complex path integral expressions for the raw moments of the Gamma exponentiated functional $\operatorname{GE}(\alpha, h)$ distribution derived in Theorem 1 by virtue of the generalized hypergeometric function ${ }_{2} F_{0}$ in the integrand. By these results a master formula is derived for raw moments which are coming from the $\mathrm{GE}(\alpha, h)$ distribution family.

As an illustrative example for $\operatorname{GE}(\alpha, h)$ distribution serves the GEEW $(\theta)$ distributed rv considered recently by Pogány and Saboor [16]. We also refer to the exhaustive list of special cases listed in Introduction.

Finally, the related probability distribution class is characterized in terms of Lambert $W$-function in Theorem 2, while the quantile function is derived in Theorem 3 in terms of the regularized upper incomplete Gamma function $Q$.

Acknowledgments: The authors are indebted to the editor and all three unknown referees for constructive comments and criticisms of an earlier draft of this paper.

Author Contributions: All authors contributed equally to this work.

Conflicts of Interest: The authors declare no conflict of interest.

\section{References}

1. Lehmann, E.L. The power of rank tests. Ann. Math. Statist. 1953, 24, 23-43; MR 0054208.

2. AL-Hussaini, E.K.; Ahsanullah, M. Exponentiated Distributions; Atlantis Studies in Probability and Statistics; Atlantis Press: Paris, France, 2015; MR 3309998.

3. Stacy, E.W. A generalization of the gamma distribution. Ann. Math. Statist. 1962, 33, 1187-1192; MR 0143277.

4. Zografos, K.; Balakrishnan, N. On families of Beta- and generalized Gamma-generated distributions and associated inference. Stat. Methodol. 2009, 6, 344-362; MR 2751078.

5. Ristić, M.M.; Balakrishnan, N. The Gamma-exponentiated exponential distribution. J. Stat. Comput. Simul. 2012, 82, 1191-1206; MR 2958781.

6. Ristić, M.M.; Nadarajah, S. A new lifetime distribution. J. Stat. Comput. Simul. 2014, 84, 135-150; MR 3169316.

7. Pogány, T.K. The exponentiated exponential Poisson distribution revisited. Statistics 2015, 49, 918-929; MR 3367731.

8. Cordeiro, G.M.; Ortega, E.M.M.; da Cunha, D.C.C. The exponentiated generalized class of distributions. J. Data Sci. 2013, 11, 1-27; MR 2963349.

9. Pinho, L.G.B.; Cordeiro, M.G.; Nobre, J.S. The Gamma-exponentiated Weibull distribution. J. Stat. Theory Appl. 2012, 11, 379-395. 
10. Castellares, F.; Lemonte, A.J. A new generalized Weibull distribution generated by Gamma random variables. J. Egypt. Math. Soc. 2015, 23, 382-390; MR 3355432.

11. Gupta, R.C.; Gupta, P.L.; Gupta, R.D. Modeling failure time data by Lehman alternatives. Comm. Statist. Theory Methods 1998, 27, 887-904; MR 1613497.

12. De Pascoa, M.A.R.; Ortega, E.M.M.; Cordeiro, G.M. The Kumaraswamy generalized Gamma distribution with application in survival analysis. Stat. Methodol. 2011, 8, 411-433; MR 2819991.

13. Cordeiro, G.M.; Nadarajah, S.; Ortega, E.M.M. The Kumaraswamy Gumbel distribution. Stat. Methods Appl. 2012, 21, 139-168; MR 2916925.

14. Pogány, T.K.; Cordeiro, G.M.; Tahir, M.H.; Srivastava, H.M. Extension of generalized integro-exponential function and its application in study of Chen distribution. Appl. Anal. Discrete Math. 2017, 11, 434-450; MR 3719836.

15. Rezaei, S.; Marvasty, A.K.; Nadarajah, S.; Alizadeh, M. A new exponentiated class of distributions: Properties and applications. Comm. Statist. Theory Methods 2017, 46, 6054-6073; MR 3620933.

16. Pogány, T.K.; Saboor, A. The Gamma exponentiated exponential-Weibull distribution. Filomat 2016, 30, 3159-3170; MR 3593064.

17. Olver, F.W.J.; Lozier, D.W.; Boisvert, R.F.; Clark, C.W. (Eds.) NIST handbook of mathematical functions; U.S. Department of Commerce, National Institute of Standards and Technology: Washington, DC, USA; Cambridge University Press: Cambridge, UK, 2010; MR 2723248.

18. Kilbas, A.A.; Srivastava, H.M.; Trujillo, J.J. Theory and Applications of Fractional Differential Equations; North-Holland Mathematical Studies; Volume 204; Elsevier: Amsterdam, The Netherlands; London, UK; New York, NY, USA, 2006; MR 2218073.

19. Dereziński, J. Hypergeometric type functions and their symmetries. Ann. Henri Poincaré 2014, 15, 1569-1653; MR 3232581.

20. Sokal, A.D. A ridiculously simple and explicit implicit function theorem. Sèm. Lothar. Combin. 2009, 61, B61Ad; MR 2529395.

(C) 2018 by the authors. Licensee MDPI, Basel, Switzerland. This article is an open access article distributed under the terms and conditions of the Creative Commons Attribution (CC BY) license (http://creativecommons.org/licenses/by/4.0/). 